\title{
Der Entwurf der neuen Psychopathengesetze für die Niederlande.
}

\author{
Von \\ J. van der Torren, \\ Nervenarzt in Hilversum (Niederlande).
}

(Eingegangen am 21. Februar 1912.)

Wie in allen zivilisierten Ländern besteht auch in den Niederlanden die Schwierigkeit, die verschiedenen Verbrecher zweckmäßig unterzubringen und bei Zumessung der Strafe oder in der Behandlung der großen Verschiedenheit gerecht zu werden, welche zwischen diesen Individuen und den Geistesnormalen einerseits, den zweifelsohne geisteskranken Verbrechern andererseits besteht. Bestand für die Kinder das Kindergesetz mit seinen verschiedenen Maßregeln, Erziehungsanstalten und Zuchtschulen, so konnte für den Erwachsenen der Richter doch nur die Wahl treffen zwischen Gefängnisstrafe und Irrenanstalt. Vor kurzem ist nun ein besonderes Strafgefängnis für die Psychopathen eröffnet, mit einem Psychiater als Direktor. Daneben hat das Justizministerium zwei Gesetzentwürfe, die „Psychopathengesetze“, der zweiten Kammer der Abgeordneten vorgelegt, welche Gesetzentwürfe auch in weiteren Kreisen Interesse erregen möchten, weshalb ich, der Bitte der Redaktion dieser Zeitschrift folgend, hier eine Ubersicht dieser Entwürfe gebe; sie ist den „Psychiatrische en Neurologische Bladen“ 15, 588, 1911 entlehnt.

Nachfolgende Erwägungen haben nun die Regierung dazu geführt, die Entwürfe der Kammer vorzulegen:

1. Welche Maßregeln müssen getroffen werden, wenn der Verbrecher zur Zeit seines Verbrechens deutlich geisteskrank und deshalb unzurechnungsfähig, zur Zeit des richterlichen Urteils wieder geheilt ist oder nicht.

Ist der Verbrecher noch nicht geheilt, so kann er in einer Irrenanstalt untergebracht werden. Dies ist aber öfter weder im Interesse der Gesellschaft, noch des Verbrechers selber, weil, sobald der in der Irrenanstalt untergebrachte Verbrecher dort geheilt ist, er entlassen werden muß. Damit ist aber die Gefahr für die öffentliche Ordnung noch keineswegs gewichen, weil öfter die Psychose rasch rezidivieren kann, 
weiter das Verbrechen mit der Psychose an und für sich nur in einem entfernten Zusammenhang stehen kann, vielmehr aus einem neben der Psychose bestehenden Grundzustand und Anlage hervorgegangen ist. Weiter besteht, auch wenn die Psychose zur Zeit des richterlichen Ausspruchs schon abgeheilt ist, und der Richter jetzt freisprechen muß, öfter eine Gefahr für ein baldiges Rezidiv dieser für die Gesellschaft äußerst gefährlichen krankhaften Zustände.

2. Viel größer sind aber die Schwierigkeiten, wenn der Verbrecher offenkundig zu der großen Gruppe der ,,vermindert Zurechnungsfähigen“ gehört. Bei Freispruch oder Unterbringung in einer Irrenanstalt bestehen in solchen Fällen die unter 1. genannten Gefahren weiter. Wird aber der vermindert Zurechnungsfähige doch zurechnungsfähig erklärt, so muß die Verbüßung einer Strafe folgen. Ist diese Strafe eine mildere, so bleibt das Rechtsgefühl öfter gänzlich unbefriedigt und es wird für die Interessen der Gesellschaft ungenügend Sorge getragen. Weiter hat bei Auflegung einer gewöhnlichen Strafe wie bei den Normalen dies öfter die Folge, daß der krankhafte Geisteszustand des vermindert Zurechnungsfähigen sich verschlimmert, weshalb andere Strafen, welche diesen Geisteszustand berücksichtigen, nötig sind. Und weiter ist dieser krankhafte Geisteszustand bei richtiger Pflege öfter verbesserungsfähig, ja sogar heilbar, aus welchen Tatsachen hervorgeht, daß es möglich sein muß, eine richtige $Z$ wangspflege aufzuerlegen. Der Justizminister meint nun in nachfolgender Weise dies erreichen zu können.

I. Erstens wünscht die Regierung nicht, die verminderte Zurechnungsfähigkeit im Gesetz niederzulegen. Sie meint, daß es genügen wird, wenn der Richter, völlig frei in seiner Abschätzung des Grades der $\mathrm{Zu}$ rechnungsfähigkeit, instand gesetzt wird, für jeden besonderen Fall aus den ihm zur Verfügung stehenden Maßregeln eine Wahl zu treffen. Ist der Verbrecher nun gänzlich unzurechnungsfähig, so kann niemals Strafe auferlegt werden, sondern nur Zwangspflege, wenn nicht schon in solchem Falle der Täter einfach in Freiheit gesetzt wird. Wird aber der Verbrecher, auch der Psychopath, vom Richter als zurechnungsfähig betrachtet, so soll er gestraft werden, und jetzt kann der Richter, wenn es ihm für den Geisteszustand des Verurteilten notwendig erscheint, eine Wahl treffen aus den besonderen zur Verfügung gestellten Strafen. Im Interesse der öffentlichen Ordnung wird es überdies möglich sein müssen, bedingt oder unbedingt, eine Zwangspflege aufzuerlegen.

II. Die Veränderungen der Strafrechtspflege. In jedem Stadium der Sache kann der Richter erklären, daß vermutet werden muß, daß der Verdächtige oder der schon Angeklagte sich während seiner Tat in einem Zustand von Störung oder mangelhaften Entwicklung seines geistigen Vermögens befand und deshalb seine eigenen Interessen 
selber nicht genügend vertreten kann. Ihm wird dann ein Ratgeber gestellt, der den Ratgebern eines minderjährigen, 16-18 Jahre alten Angeklagten gleichgesetzt wird, während der Angeklagte selber mit solch einem minderjährigen Angeklagten gleichgesetzt wird. Weiter wird es möglich sein, den Angeklagten zur Beobachtung in einer Heilanstalt, aber keiner Irrenstalt, unterzubringen, während einer höchstens sechswöchentlichen Zeitdauer. (Bis auf heute mußte die sachverständige Untersuchung immer im Gefängnis stattfinden.)

III. Drittens wünscht die Regierung die auferlegte Strafe auf der einen und die Maßregeln im Interesse der Gesellschaft auf der anderen Seite strengstens voneinander geschieden zu halten. Also eine strenge Scheidung zwischen Strafe als Vergeltung von Schuld und Behandlungsmaßregeln. Utber die Art der Strafe verfügt deshalb der Strafrichter, auch wird er bestimmen, ob der schuldig Erklärte bedingt oder unbedingt zur Verfügung der Regierung (,,ter beschikking der regeering ") gestellt werden wird. Der Zivilrichter bestimmt dann die Art der Behandlungs maßregeln und ihre Zeitdauer.

Strafen. Bei der dem Richter vollkommen überlassenen Freiheit in der Wahl der Strafe wird es deshalb auch möglich sein, dem Psychopathen eine Strafe aufzuerlegen, derjenigen gleichgesetzt, welche auch dem Normalen auferlegt werden kann. Hier wird auch noch die bedingte Nichtvollstreckung der Strafe eingeführt.

Neben den gewöhnlichen befinden sich aber die für den Psychopathen besonderen Strafen. Die gewöhnlichen Strafen sind: Gefängnisstrafe, Verhaftung und Geldbuße. Unter dem Alter von 18 Jahren kann statt der Hauptstrafe auferlegt werden: Unterbringung in einer Zuchtschule (tuchtschool), Geldbuße oder Verweis (berisping).

Als besondere Strafen für Psychopathen werden jetzt im Entwurf genannt:

1. Unterbringung in einem besonderen Strafgefängnis,

2. Geldbuße,

3. mündlicher oder schriftlicher Verweis.

Die Dauer der Unterbringung im besonderen Strafgefängnis beträgt wenigstens eine Woche, höchstens die Dauer der Strafe für Normale in einem gewöhnlichen Strafgefängnis.

Die Geldbuße wechselt zwischen fl. 5. - und fl. 6000.-. Sie kann nur auferlegt werden, wenn für das Verbrechen eine Gefängnisstrafe von höchstens 4 Jahren oder Verhaftung gefordert wird. Bei Nichtbezahlung folgt Unterbringung in einem besonderen Strafgefängnis für die Zeitdauer von 1 Woche bis zu 1 Jahre.

Ein Verweis erfolgt z. B. bei leichteren Verbrechen, wenn die Hauptstrafe für Normale nur in einer Geldbuße oder Haft besteht.

Für die Auferlegung der besonderen Strafen muß der Richter da- 
von überzeugt sein, daß das Krankhafte der geistigen Veränderung absolut sicher wissenschaftlich feststeht. In bezug auf echte moralische Defekte und gewisse sexuelle Perversitäten und im allgemeinen auf solche Charakterfehler und Mängel, ,welchen meistens die verbrecherische Natur eines Verbrechers zuzuschreiben ist", steht das nicht fest.

Zwangs pflege. Neben den psychopathischen Verbrechern können auch unter im Entwurf festgelegten Umständen die Störung verursachenden Potatoren, die Bettler und Landstreicher nach der ihnen wohl oder nicht auferlegten Strafe vom Richter zur Verfügung der Regierung gestellt werden. Der Zivilrichter untersucht nun wieder die Sache. In Ubereinstimmung mit dem Ausspruch des Strafrichters: bedingte oder unbedingte Zurverfügungstellung der Regierung, bestimmt der Zivilrichter die Dauer der Zwangspflege (1-5 Jahre, wenn die Missetat oder Úbertretung geschehen ist entweder mittels der Presse oder unabsichtlich; 2-10 Jahre wegen eines oder mehrerer anderer Verbrechen). Bei der unbedingten Zurverfügungstellung kann der Zivilrichter den geisteskranken, nicht zurechnungsfähigen Verbrecher vorlä ufig für die Zeitdauer von höchstens 1 Jahre in einer Irrenanstalt unterbringen lassen.

Bei der bedingten Zurverfügungstellung werden vom Zivilrichter die Bedingungen festgestellt und eine Probezeit von höchstens 5 Jahren.

Die Zeitdauer der Zwangspflege kann, wenn nötig, jedesmal während einer Zeitdauer von $1 / 2-2$ Jahren verlängert werden, wobei sachverständige Belehrung eingeholt wird.

Der zur Verfügung der Regierung gestellte Verbrecher kann nun untergebracht werden:

1. in einem Reichsasyl, zu welchen ein gesondertes Asyl für Trunksüchtige gehören soll. In diesem Asyl können nachfolgende Strafen auferlegt werden:

a) Verbot von Schreiben oder Empfang von Briefen, von Besuch oder von anderen Vorrechten.

b) Darreichung von Wasser und Brot statt der gewöhnlichen Nahrung.

c) Einsperrung im Cachot.

d) Fesseln.

Die ärztliche Behandlung wird hierbei jedoch im Vordergrund stehen müssen, und daneben religiöse Auferziehung und Unterricht, gewöhnlicher Unterricht (einschließlich fachmännischem Unterricht) und Arbeit.

Im Falle von Geisteskrankheit, ernstlichen oder Infektionskrankheiten oder Schwangerschaft können die Ieute in einer Irrenanstalt respektive anderen Anstalten untergebracht werden. 
2. Zwangspflege in privaten Vereinigungen und Anstalten.

3. Familienpflege für solche Leute, welche für eine Zeitdauer von wenigstens 6 Monaten in einem Reichsasyl untergebracht waren.

Entlassung. Diese kann zu jeder Zeit, bedingt oder unbedingt, vom Justizminister befohlen werden. Die bedingte Entlassung kann jederzeit aufhören, wenn die Person in einer Irrenanstalt aufgenommen werden muß, sich schlecht beträgt, für die öffentliche Ordnung gefährlich wird, den Bedingungen nicht nachkommt oder dies anderweitig nötig sein wird.

Für minderjährige Psychopathen (unter 18 Jahren) bleiben im allgemeinen die Kindergesetze gültig. Doch wird es auch möglich sein, einen sehr gefährlichen Minderjährigen in einem Reichsasyl und für einen längeren Zeitraum als bis zu seinem 21. Lebensjahre unterzubringen.

Ehe nun diese Gesetzentwürfe zu definitivem Gesetz werden, wird gewiß noch vieles in demselben abgeändert werden. Viele Wünsche sind schon rege geworden, und einer der wichtigsten ist wohl, die ganze Sache vom Strafrichter beenden zu lassen und sie nicht wieder dem Zivilrichter in die Hände zu legen, welch letzterer dann wieder von vorn anfangen muß, sich in die ganze Sache einzuarbeiten. Weiter: Zurverfügungstellung und $Z$ wangspflege unabhängig zu machen von der Schuld im strafrechtlichen Sinne des Verbrechers, damit auch in Fällen von Geisteskrankheit, wenn wegen Schuldmangels freigesprochen werden muß, Zwangspflege auferlegt werden kann. Im Entwurf doch ist der Meinung Ausdruck gegeben: keine Zwangspflege usw. ohne Schuld und Strafe. Abgesehen von Kleinigkeiten, muß man es aber begrüßen, daß die Sache endlich von der Regierung in die Hand genommen worden ist und ein Gesetzentwurf entstanden ist, der es dem Richter möglich macht, dem individuellen Geisteszustand des Verbrechers in so außerordentlich mannigfacher Weise Rechnung zu tragen. 